\title{
EL NUEVO CONTEXTO DE LAS CAMPAÑAS ELECTORALES: EL CASO DEL APP MIVOTOHOY
}

\section{POLITICAL CAMPAIGNS, CITIZEN PARTICIPATION AND THE POSSIBILITIES OF THE WEB 2.0}

\author{
Carolina Carazo Barrantes ${ }^{l}$ \\ carolina.carazo@ucr.ac.cr
}

Fecha de recepción: 11 mayo 2016 - Fecha de aceptación: 14 diciembre 2016

\begin{abstract}
Resumen
Este artículo analiza el caso de una aplicación (MiVotoHoy) propuesta al Tribunal Supremo de Elecciones (TSE) de Costa Rica para la campaña presidencial de febrero 2014 y que el TSE no autorizó. En el artículo se discute el nuevo contexto en el que hoy en día se desarrollan las campañas electorales, uno en el que las encuestas enfrentan cada vez más limitaciones para seguir cumpliendo su función de predicción en periodos electorales; al tiempo que las tecnologías de la información y la comunicación han puesto sobre la palestra temas como la conectividad móvil, el empoderamiento ciudadano y la participación política de jóvenes a través de "apps", entre otros aspectos.

Palabras clave: Comunicación política, Apps móviles, Participación política, Millennials, Encuestas políticas
\end{abstract}

\begin{abstract}
This article analises the case of an app (MiVotoHoy) proposed to the Supreme Electoral Tribunal (TSE) of Costa Rica for the presidential campaign in February 2014 which the TSE did not authorize. The article explores the new context in which today's election campaigns take place, one in which polls face increasing limitations to continue to fulfill their function of forecasters in election periods while, in the meantime, the technologies of information and communication have brought to the forefront issues such as mobile communication, citizen empowerment, political participation of youth through apps, among others.
\end{abstract}

Key Words: Political communication, Mobil apps, Political participation, Millennials, Political polls.

\section{Introducción}

La elección presidencial de los Estados Unidos realizada el 8 de noviembre 2016 fue, por muchas razones, como subirse a una montaña rusa, y no solamente por el resultado de la contienda. Quienes siguieron de cerca la cobertura mediática - los pronósticos, primero, y los resultados, después - se llevaron no pocas sorpresas. Si bien la más grande de todas fue ver al señor Trump como presidente

1 Profesora en la Escuela de Ciencias de la Comunicación Colectiva (ECCC) e investigadora del Centro de Investigación en Comunicación (CICOM) de la Universidad de Costa Rica. 
electo la madrugada del 9 de noviembre, los resultados inesperados estuvieron a la orden del día desde la temporada de elecciones primarias (en el primer semestre del año) cuando cada uno de los estados realizó elecciones para escoger a los candidatos de los partidos Demócrata y Republicano.

Por mencionar solamente algunas de las sorpresas de la temporada de elecciones primarias: el 3 de mayo de 2016 el reconocido estadístico Nate Silver, fundador del sitio web FiveThirtyEight (fivethirtyeight.com), calculó que Hillary Clinton tenía un 90 por ciento de probabilidades de ganarle a Bernie Sanders en Indiana. Sanders ganó ese estado con un confortable margen de 5\% de los votos (Rutenberg, 2016, mayo 5, parra. 3). Lo mismo había sucedido en Michigan el 8 de marzo anterior: todas las encuestas pronosticaban una incuestionable victoria para Clinton (quien estaba entre 10 y 20 puntos arriba), pero Sanders ganó por 1.5\% de los votos (National Public Radio, 2016, marzo 9, parra. 2).

Es bien conocido que el fenómeno se repitió durante las semanas y días previos al 8 de noviembre. Desde el mes de junio, luego de las convenciones de ambos partidos donde quedaron electos como candidatos Hillary Clinton y Donald Trump, el prestigioso periódico The New York Times llevaba el pulso de la elección con un modelo que indicaba, según datos de diferentes encuestas de todo el país, cuántas probabilidades de ganar tenía cada candidato (Katz, 2016, noviembre 8). El primero de junio, Clinton arrancó con 58\% de posibilidades de ganar, contra 42\% de Trump; para finales de ese mes, las posibilidades de la primera candidata mujer eran de $78 \%$ (contra $23 \%$ del republicano). Los números fueron oscilando entre $70 \%$ y $90 \%$ para Clinton y entre $30 \%$ y $10 \%$ para Trump. El propio martes 8 de noviembre, al iniciar la noche, luego de que se cerraron los primeros centros de votación en la costa este de los Estados Unidos, The New York Times informaba que Hillary Clinton tenía 85\% de posibilidades de ser electa la primera mujer presidenta de los Estados Unidos esa noche (Katz, 2016, noviembre 8).

¿Por qué fallaron las encuestas en sus predicciones? ¿Cómo fue que un candidato como Donald Trump, que parecía más bien un mal chiste y que fue tratado como tal por un importante sector de los medios norteamericanos y el mundo, resultó, primero, el candidato ganador en un partido con la trayectoria del partido Republicano y luego, contra todos los pronósticos, Presidente de los Estados Unidos?

No hay una respuesta correcta, ni sencilla: la responsabilidad de los medios y de los periodistas ha sido señalada por muchos (Kristof, 2016, marzo 26; Scarry, 2016, marzo 5); la cultura de realitytelevision o, como Vargas Llosa la llamó "la civilización del espectáculo" es el telón de fondo; incluso la cúpula del mismo partido Republicano tuvo su cuota de responsabilidad por la manera como había estado haciendo política en los últimos años (Ulibarri, 2016, mayo 8, parra. 9). Al hablar de los pronósticos equivocados de las encuestas, Rutenberg (2016, mayo 5, parra. 4) menciona los nuevos retos en las encuestas telefónicas: por un lado, la imprevisibilidad de un electorado enojado y, por otro lado, la creciente cantidad de estadounidenses —especialmente los jóvenes-que no disponen de teléfonos fijos y son por lo tanto difíciles de localizar.

El caso de las elecciones estadounidenses del año 2016 es uno más que viene a sumarse a otros que han demostrado que estamos ante una nueva era que demanda repensar las campañas políticas tal y como se conocían hasta hace unos pocos años. Las tecnologías de información y comunicación, el uso generalizado de teléfonos inteligentes, el acceso masivo a internet y la popularidad de redes sociales como Facebook y Twitter han venido a revolucionar, entre otros muchos ámbitos de nuestras sociedades, a las campañas electorales y la participación política. No cabe duda de que se está ante una nueva forma de hacer política (tanto para los actores políticos como para la ciudadanía).

En este nuevo contexto político y tecnológico, se analiza el caso particular de la aplicación ("app" por el diminutivo de la palabra en inglés, "application") MiVotoHoy propuesta al Tribunal Supremo de Elecciones (TSE) de Costa Rica para la campaña presidencial del año 2014 y la resistencia institucional del TSE para reconocer esta nueva realidad.

El artículo explica en qué consiste la aplicación y presenta, en detalle, los argumentos del TSE para no hacer reformas en la normativa electoral y así permitir su operación. Además, se detiene a analizar el nuevo contexto en relación con la ciudadanía contemporánea y la participación de ésta en 
política. Se plantea la interrogante de cómo la web y las redes sociales han cambiado la forma como se es ciudadano; el papel que tiene la generación de los llamados "millenials", su uso de teléfonos inteligentes y de "apps", entre otros.

\section{El caso costarricense: elección presidencial de febrero 2014}

En Costa Rica, dos días después de las elecciones presidenciales del domingo 2 de febrero de 2014, el periodista Cristian Cambronero (2014) publicó en su blog, fusildechispas.com un post que tituló: Las encuestas ya fueron. El sorpresivo resultado en el que "el candidato del margen de error" en las encuestas que se habían publicado durante la campaña electoral resultó ganador de la primera vuelta, le dieron pie para sentenciar que "las encuestas de intención de voto, como las conocimos hasta ahora, están enterradas. La nueva realidad social, las dinámicas de interacción, y los nuevos espacios de formación de opinión, las mataron" (parra. 1).

Cambronero (2014) aclara que no cree que las encuestas "se equivoquen", pero sí denuncia que "su metodología es anacrónica y su publicación es siempre tardía" (parra. 2).

Esta no es, sin embargo, una reflexión enfocada en las encuestas. A pesar de su contundencia, lo que llama la atención del texto de Cambronero es, por un lado, su propuesta: enmarcada en la nueva era, la era de las “apps", de la conectividad móvil, de la interactividad, del empoderamiento ciudadano y, por otro lado, la posición del Tribunal Supremo de Elecciones (TSE) de cerrarle las puertas a la innovación y actuar como si esta nueva realidad no estuviera aquí para quedarse.

\section{MiVotoHoy: una posibilidad adicional de información en época de campaña}

En setiembre de 2013, la agencia Big Web Noise, de la cual Cambronero es socio fundador, lanzó la aplicación MiVotoHoy, una aplicación que permitía registrar, en tiempo real, la intención de voto de los usuarios de teléfonos con sistema operativo iOS o Android en los cuales se instalaba.

Cada usuario contaba con su propio voto, el cual podía modificar para uno u otro candidato cada vez que quisiera. La idea era llevarle el pulso a la intención de voto y, sobre todo, a los cambios que presentaría a lo largo del tiempo como resultado de los distintos acontecimientos de la actualidad. Es decir, se trataba de una herramienta que proveería de información riquísima para analizar cómo los acontecimientos de la actualidad nacional (los debates televisivos, la publicación de las encuestas, las renuncias de precandidatos y candidatos, entre otros eventos) impactan las preferencias de los votantes en tiempo real. Los resultados del sondeo permanente estaban accesibles de forma ininterrumpida, por medio de la "app", o de la web y visibles para cualquier persona. Precisamente, en declaraciones al periódico La Nación cuando fue lanzada la aplicación, Cambronero había dicho: "Esta herramienta nos permite apreciar cómo los hechos de la actualidad inciden en la intención de voto de la gente; pero también cómo puede la gente usar su intención de voto para incidir en la campaña política" (en Ruiz, 2013, septiembre 21, parra. 6).

La aplicación era gratuita y podía ser descargada en el AppStore de Apple o el Play Store de Google. También se podía tener acceso a ella vía web por medio del sitio MiVotoHoy.com. 
Figura 1

Capturas de pantalla de MiVotoHoy (setiembre-octubre 2013)
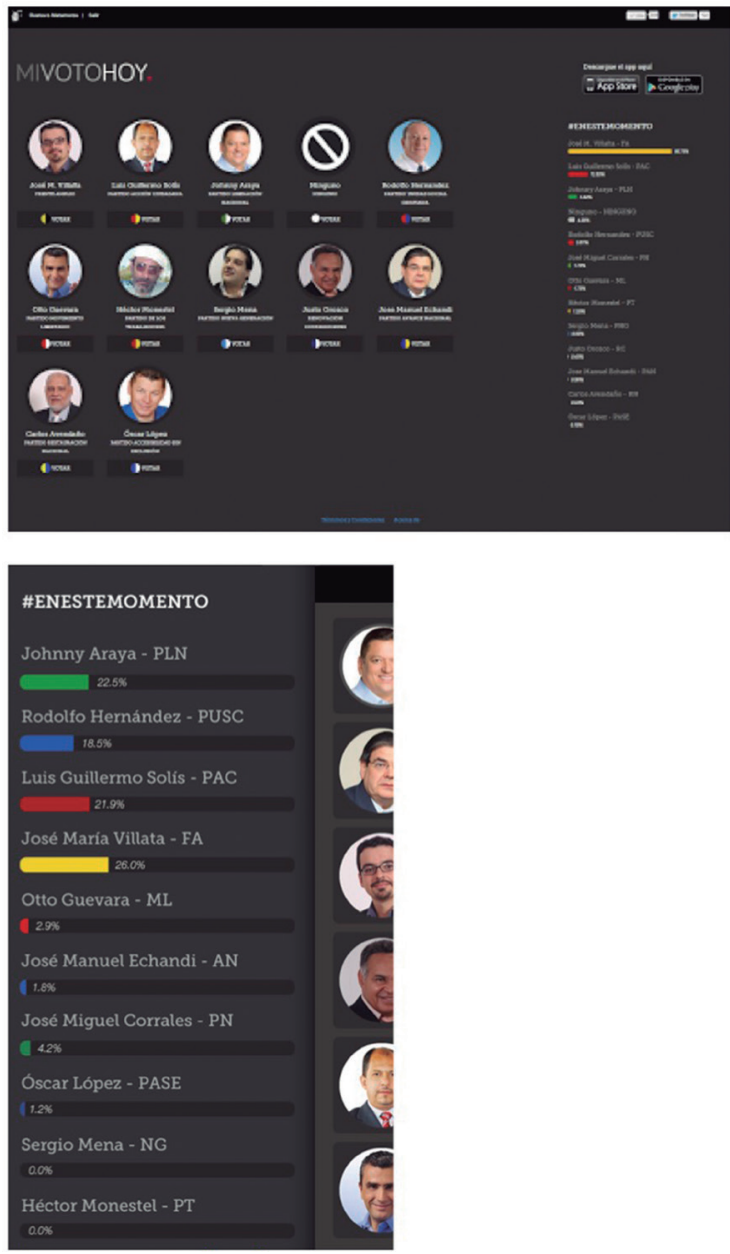

Fuentes: www.mivotohoy.como y https://play.google.com/store/apps/details?id=com.bigwebnoise.mivotohoy

MiVotoHoy nació a partir de la lectura que hizo la agencia Big Web Noise de una realidad indiscutible: en la actualidad, la opinión y la intención de voto de los electores puede variar rápidamente. "No existen ya los arraigos partidarios de antaño, las lealtades a muerte, ni mucho menos los cheques en blanco. Las encuestas tradicionales son incapaces de recoger esos cambios" (Cambronero, 2014, febrero 4, parra. 10).

Además, como bien apuntó el director de desarrollo de la agencia, Allan Naranjo: "esta plataforma le permitirá a la gente participar de la campaña política usando las herramientas a las que ya está acostumbrada" (en Ruiz, 2013, septiembre 21, parra. 7).

Se debe tener en cuenta que entre el 21 de septiembre y principios de octubre, cuando la "app" fue retirada por disposiciones del TSE (como se verá a continuación), había sido descargado por más de 15 mil usuarios (Cambronero, 2014, febrero 4, parra. 5). Eso sin que la campaña política hubiera ni siquiera calentado. 


\title{
La respuesta del TSE
}

El artículo 138 del Código Electoral obliga a institutos, universidades, cualquier ente público o privado y las empresas dedicadas a elaborar encuestas y sondeos de opinión de carácter político-electoral a registrarse ante el TSE. Existe, por tanto, un reglamento sobre la inscripción para la realización de encuestas y sondeos de opinión de carácter político-electoral (Tribunal Supremo de Elecciones, 2009). El reglamento exige, entre otros:

- Aportar todos los documentos necesarios que evidencien la experiencia del interesado en la elaboración de encuestas y sondeos de opinión (Art. 3, inciso d)

- Aportar una certificación extendida por el Colegio de Profesionales en Ciencias Económicas, en la cual se acredite que la empresa se encuentra debidamente registrada y los profesionales incorporados al mismo (Art. 3, inciso g)

\begin{abstract}
- Que cuando se difunda o publique una encuesta o sondeo la empresa guarde y tenga a disposición del TSE todos los documentos que respalden el trabajo, tales como: ficha técnica que incluye la cobertura geográfica, el tipo de muestreo, tamaño de la muestra, error de muestreo, nivel de confianza, selección de la muestra, controles de calidad, fechas en que se realizó el trabajo de campo, entre otros (Art. 7).
\end{abstract}

Queda claro que ni la metodología de sondeo de la "app", ni la naturaleza independiente y ciudadana de la plataforma, ni el perfil no-estadístico de la empresa que lo desarrolla cumplían con lo que establece el Reglamento del TSE. Los creadores del app enviaron, oportunamente, antes del inicio de la campaña (en agosto 2013), una carta al TSE para solicitar que se modificara el reglamento de manera que iniciativas innovadoras como MiVotoHoy pudieran ser legítimas a los ojos del rector del proceso electoral.

En la carta de justificación Cambronero advierte: "bajo el presente reglamento no es posible el registro y regularización de MiVotoHoy o proyectos similares; pero en caso de hacerse públicos, sí aplicaría el reglamento para sancionarlos" (Cambronero, 2013, agosto 8. Comunicación personal. Carta a Héctor Fernández Masís, Director General del Registro Electoral y de Financiamiento de Partidos Políticos. Parra. 18).

El TSE analizó el caso y comunicó a la agencia que estimaba "improcedente modificar la reglamentación de mérito, cuyas normas son suficientemente claras y que, por ello, no demandan aclaración o precisión adicional por parte de sus Magistrados” (Tribunal Supremo de Elecciones, 2013, septiembre 5, parra. 2).

En una comunicación interna del TSE (2013, agosto 29), Héctor Fernández Masís, encargado de la Dirección General del Registro Electoral y de Financiamiento de Partidos Políticos detalló su criterio:

Las regulaciones vigentes en la legislación electoral sobre esta materia, pretenden que las encuestas o sondeos de opinión de carácter político-electoral, que se elaboren, publiquen y difundan durante el proceso electoral, que podrían tener un impacto sobre la opinión pública o el electorado, se realicen con base en técnicas y metodologías estadísticas, que permitan garantizar la representatividad de los resultados y, por otro lado, que ante cualquier cuestionamiento o denuncia presentada por un tercero, se pueda corroborar la autenticidad de la información (p. 4).

El análisis de la argumentación deja ver que al TSE le preocupaba, ante todo, que la "app" tuviera fines no-estadísticos y resultados ilustrativos sin representatividad estadística, lo cual no le permitiría garantizar: 
- que se trata de resultados representativos de la población empadronada.

- la comparabilidad de los resultados obtenidos respecto a otros estudios de opinión que apliquen metodologías estadísticas.

- que las personas que ingresen al sitio web a consultar los resultados del sondeo o que hayan participado del mismo, estén conscientes de que no se trata de un sondeo o encuesta metodológicamente científica, cuyos resultados podrían verse afectados por factores como los señalados en los puntos anteriores (TSE, 2013, agosto 29, p. 8).

Adicionalmente, el TSE manifestó preocupación porque al variar la reglamentaciónde manera que se autorice la inscripción de empresas que realicen sondeos con herramientas informáticas como la propuesta, pondría en desventaja y desigualdad de condiciones a quienes aplican metodologías científicas y estadísticas para la elaboración y desarrollo de los estudios de opinión, que en principio pretenden garantizar o respaldar la autenticidad de la información obtenida. Además se generaría una desigualdad en cuanto a los costos en que incurren precisamente para garantizar la confiabilidad de esa información (...) (TSE, 2013, agosto 29, p. 8).

Así, el TSE dejó vigentes las normas y requisitos pensados solo para regular encuestas tradicionales. "La decisión dejó a MiVotoHoy en ese limbo legal en el que cae lo que no está prohibido pero tampoco formalmente permitido" (Cambronero, 2014 febrero 4, parra. 5).

\section{Cuatro razones que exigen un cambio}

Entre muchas razones que podrían esgrimirse para presentar argumentos a favor de que el TSE levante su ancla del pasado y navegue hacia el presente, ni qué decir, hacia el futuro, se pueden precisar cuatro: 1) una ciudadanía más informada y crítica; 2) la web y las redes sociales cambiaron la manera en la que se es ciudadano; 3) la nueva era es de los "millennials" y las "apps" y 4) las encuestas son valiosas pero tienen limitaciones.

\section{Ciudadanía más informada y crítica}

Estudiosos y analistas coinciden que estamos frente a una ciudadanía cuyo comportamiento electoral es distinto. En palabras de Sergio Araya (2014, febrero 4), coordinador de proyectos del área política de la Fundación Konrad Adenauer, el electorado "parece ir migrando paulatinamente hacia criterios más racionales y menos emotivos para definir su voto" (parra. 19).

El estudio poselectoral del Centro de Investigación y Estudios Políticos (CIEP) de la Universidad de Costa Rica presenta datos que dan testimonio de este cambio. Al indagar — en una pregunta cerrada- sobre la relevancia de fuentes de información en la decisión de voto para presidente, los debates son los que mayor número de personas consideró que tomó mucho en cuenta (65\%). Los programas de opinión de la televisión también fueron relevantes, ya que $49 \%$ dijo haberlos tomado mucho en cuenta. En cambio, las redes sociales (Facebook y Twitter) no fueron para nada importantes para el 53\% de los electores (CIEP, 2014, p. 32).

Al preguntarse, de forma abierta, cuál fuente de información influyó más en la decisión, la fuente más citada fueron los debates (33\%), seguidos por los programas de opinión y noticias (21\%). Solo un $5.4 \%$ respondió que Internet y redes sociales como Facebook y Twitter influyó en su decisión.

El mismo presidente del TSE, Luis Antonio Sobrado, reconoció, la noche en que se dio a conocer el primer corte con los resultados de la segunda ronda el 6 de abril de 2014, que estamos ante una ciudadanía más informada y madura: 
[...] nuestro pueblo respondió con una madurez cívica impresionante. Esta Costa Rica políticamente adulta, que cambió las pitoretas por las preguntas directas y el confeti por el reclamo frontal, transitó estos últimos meses entre incertidumbres y acertijos políticos". (...) Este país estuvo a la altura del desafío. Al hambre de información de un elector que hoy decide más con su cabeza (menos con su corazón y nunca con las vísceras), la prensa respondió con múltiples debates, amplia cobertura periodística y plataformas digitales de análisis de las propuestas de gobierno (Sobrado, 2014 abril 6, pp. 1-2).

No cabe duda de que los costarricenses pueden reconocer la diferencia entre datos de una aplicación del celular que no son representativos y los de una encuesta hecha por reconocidos institutos, universidades o empresas encuestadoras y publicados o difundidos por medios de comunicación.

Sí puede, y debe, exigir el TSE que cualquier plataforma o aplicación innovadora que no esté amparada por una metodología que asegure resultados representativos, así lo aclare de manera visible y clara. O, incluso, en una primera etapa (que podría considerarse "de transición") podría exigir un muestreo representativo y sistematización en el tratamiento de los datos para, idealmente, llegar a un escenario en el que se pueda aprovechar la imperante conectividad móvil para contar con instrumentos capaces de registrar los cambios en la intención de voto en tiempo real.

\section{La web y las redes sociales cambiaron la manera como somos ciudadanos}

La Internet y las redes sociales ya no son nuevas y mucho se ha escrito sobre su impacto en la ciudadanía. Rueda Ortiz (2009), Sierra Caballero (2013), Bernete García (2013) y Alcoceba Hernando (2013) son tan solo algunos de los autores que han escrito sobre el tema de tecnología y ciudadanía en años recientes. Estos autores hablan de conceptos como "ciudadanos digitales" y "ciberciudadanía".

Para Rueda Ortiz (2009), la manera tradicional de concebir la política puede sobrevivir y traslaparse por más tiempo, pero es evidente que las nuevas prácticas ya no se pueden ignorar y exigen entender la ciudadanía mucho más ampliamente que restringida a la acción política de partidos, al voto y a la esfera pública del periódico y de las noticias: "El ejercicio ciudadano requiere entenderse como una práctica, no necesariamente circunscrita a los espacios instituidos formales y tradicionales de participación política" (p. 9).

Cambronero lo planteó de la siguiente manera en la carta que le envió al TSE para procurar abrirle paso a MiVotoHoy:

Las tecnologías de información y comunicación ya han alterado de forma dramática la forma en que los ciudadanos accedemos a la información, la replicamos y la redistribuimos. También han impactado las posibilidades reales de incidencia que la gente común tiene sobre la función pública y el acontecer nacional. Pocas adiciones han estimulado la participación democrática legítima y el ejercicio de la ciudadanía desde nuevas plataformas, como la internet y las comunicaciones móviles. Consideramos que la legislación y los reglamentos vigentes del TSE deben estar a la altura de los tiempos y adaptarse a un entorno de innovación en el que nuevos actores adquieren un papel protagónico, e introducen novedades que - no es demasiado ambicioso creerlo-cambiarán la forma en que los ciudadanos viven el proceso democrático: en tiempo real y desde la palma de la mano (Cambronero, 2013, agosto 8. Comunicación personal. Carta a Héctor Fernández Masís, Director General del Registro Electoral y de Financiamiento de Partidos Políticos. parra. 22).

En la campaña electoral hacia las elecciones presidenciales de febrero (y abril) 2014, no sólo se vivió y se sintió, sino que hubo mediciones que no dan cabida a dudas de que en Costa Rica se replica el fenómeno que se ha estudiado en otros contextos.

Según el estudio poselectoral del CIEP (2014), más personas participaron en redes sociales como Facebook o Twitter (31.8\%) que quienes pusieron banderas o calcomanías en la casa o en el carro (19.5\%), participaron en reuniones de discusión y análisis (12.3\%), participaron en plazas públicas, encuentros ciudadanos, caravanas (10.0\%) y quienes participaron en actividades el día de las elecciones, como transporte, guías o fiscales (8.3\%) (p.7). 
Dos notas publicadas por el diario La Nación inmediatamente después de la primera ronda también ilustran el papel de las redes sociales: el lunes 3 de febrero, en la edición digital, el titular de la noticia era: Google, Twitter y Facebook anunciaron despegue de Luis Guillermo Solís en intención de voto (Fonseca, 2014 febrero 3) y el martes 4 se le dio seguimiento al tema con la noticia en la versión impresa: Redes sociales anunciaron cómo se resolvió elección presidencial en Costa Rica (Lara, 2014 febrero 4).

Es interesante recordar que estas notas, que fueron publicadas luego de la primera fecha electoral, no fueron las únicas dos notas que este influyente diario dedicó al tema, pues ya había publicado varias notas sobre el comportamiento de los candidatos en redes sociales. Esto no es de extrañar puesto que las redes sociales y sus tendencias se han convertido en tema de agenda noticiosa y se ha hecho cada vez más común en este y otros medios impresos, digitales, televisivos y radiofónicos reportar sobre las tendencias que se ven reflejadas en sitios como Facebook y Twitter, las redes sociales más populares en Costa Rica. Así, el 24 de enero, ocho días antes de la elección, La Nación dedicó una página completa a una nota titulada: En Facebook, Luis Guillermo Solís genera más movimiento comparado a rivales. La nota incluía una bajada (subtítulo) destacado que leía: Político aumentó 96\% adhesiones a página y sumó más interacción en 53 días (ver Figura 2) y, en la versión en línea, una aclaración, resaltada en formato de bajada (ver Figura 3) con el siguiente texto:

Este estudio no refleja el respaldo para la elección del 2 de febrero, como lo haría una encuesta de opinión pública pero sí describe tendencias extraídas de millones de cifras, ligadas a los perfiles de estos políticos en una plataforma social usada a diario por más de un millón de personas solo en la Gran Área Metropolitana (Lara, 2014 febrero 24).

Figura 2

Nota en el periódico La Nación del 24 de enero 2014
Figura 3

Versión en línea del 24 de enero 2014

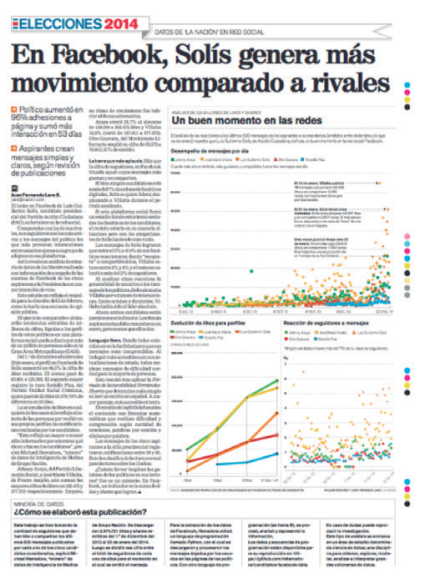

Fuente: La Nación, 24 de enero de 2014.

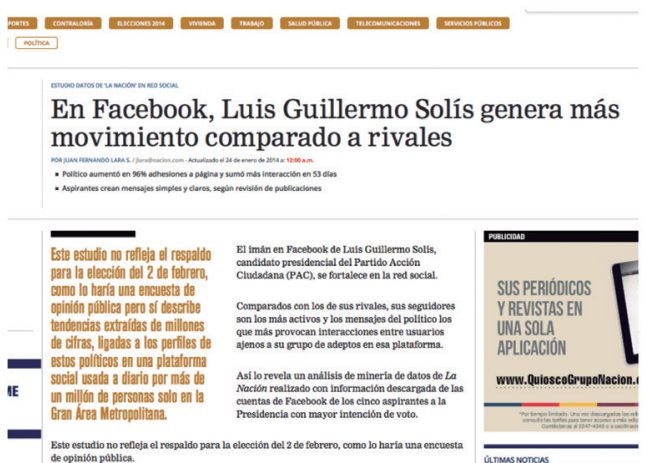

Fuente: http://www.nacion.com/nacional/política/Facebook-Solismovimiento-comparado-rivales-0_1392460782.html

¿Por qué extenderse en tanto detalle sobre esta nota? Simplemente porque cabe la pregunta: ¿en qué medida es la publicación de estos resultados diferente de lo que pudieron haber sido los resultados de la "app" MiVotoHoy? Pareciera que no tanto.

En el año 2015, el politólogo Roberto Cruz Romero publica, en el reconocido informe del Programa de la Sociedad de la Información y el Conocimiento (PROSIC) de la Universidad de Costa Rica, un estudio titulado Política 2.0: Las TIC en la campaña electoral presidencial 2014 en Costa Rica. El autor sostiene que: 
el portillo se ha abierto en relación a las nuevas formas de hacer política, de mano de la tecnología. Internet, como base de esta nueva tendencia, representa un campo de acción prácticamente libre para ciudadanos e instituciones, políticas y estatales, donde confluyen viejos y nuevos modelos de estructuración social, comunicativa y de poder (Cruz Romero, 2015, p. 295).

\begin{abstract}
Si bien no es la posición del presente artículo proponer que los datos de una "app" como MiVotoHoy puedan acercarse siquiera a datos estadísticos representativos, Cruz Romero (2015), luego de hacer un interesante y pormenorizado estudio del uso de las redes sociales durante la campaña electoral 2014, sí se acerca a esa posición al afirmar en sus conclusiones que "el caso de Costa Rica ha demostrado que es posible tener información confiable de Internet, específicamente de las redes sociales, y en caso más concreto Facebook. Ello habla de una representatividad social (o estadística) cada vez más significativa” (p.330).
\end{abstract}

Para el autor, la creciente cantidad de gente que participa en ese mundo virtual hace que cada día se acerque más al mundo real:

Cada día existen más conexiones y más gente viviendo parte de su vida online, con lo que, a pesar de la brecha digital existente, puede esperarse una dinámica que refleje de manera fiel las problemáticas y debates sociopolíticos del mundo real en espacios virtuales de interacción. Ello apunta a considerar las redes sociales como fuente confiable de información y datos. No es necesario que "toda" la gente esté conectada, dado que tal indicador crecerá con el tiempo, sino la forma en que se interpreta lo que dicha gente diga o haga (Cruz Romero, 2015, p. 330).

\title{
3. La nueva era es de los millennials y las "apps"
}

Los datos del propio TSE dirigen el foco hacia la creciente importancia de la generación "Y", o "los millennials", como se le conoce a la generación del milenio (jóvenes nacidos entre 1981 y 19972000); es decir, personas que en el año 2015 tenían entre 18 y 34 años. En las elecciones municipales de febrero de 2016, según los datos de cierre del Padrón Nacional Electoral (Tribunal Supremo de Elecciones, 2015, noviembre 18), de los 3,2 millones de electores, el 50\%, es decir, cerca de 1,6 millones de votantes, tenía entre 18 y 39 años. Si se considera solo a los menores de 34 años, el peso de esta población en el padrón era del $40 \%$.

En esas elecciones, no solamente eran el segmento de la población más importante en el Padrón Electoral, sino que, según un estudio del Centro de Investigación y Estudios Políticos (CIEP) de la Universidad de Costa Rica (UCR) el 77\% de los ciudadanos de entre 18 años y 34 años expresó intención de votar, un porcentaje siete puntos mayor que en la población general. El 85\% de quienes podían votar por primera vez en este tipo de elecciones lo pensaba hacer (Murillo, 2016, enero 13, parra. 3 y 5).

Al mismo tiempo, se trata del grupo de edad más enchufado en la era de los dispositivos conectados. Según un estudio de Nielsen (2014, septiembre 5), el 85\% de las personas entre los 18 y 34 años posee un smartphone o teléfono inteligente. Es muy probable que esta cifra haya aumentado aún más desde el año 2013 en que Nielsen realizó el estudio.

En Costa Rica, según datos de la Superintendencia de Telecomunicaciones (Sutel), la población está conectada a Internet, principalmente mediante smartphones (en Cordero, 2014, mayo 25, parra. 1). Datos de la Sutel revelan que:

al primer semestre del 2013 había 3.990.656 suscriptores de Internet (fijo y móvil) para una población de 4,3 millones de personas, según el Censo del 2010. Llegar a esta cantidad de conexiones implicó un crecimiento del $72 \%$ en solo 18 meses.

Este crecimiento fue impulsado principalmente por Internet móvil: el acceso a redes sociales, consumo de contenido (video) y búsqueda de información en la Red con teléfonos inteligentes. 
De esta manera, del total de conexiones de Internet a junio del 2013, un 88\% corresponden a suscripciones de Internet móvil, es decir, servicios que se prestan mediante una red celular $3 \mathrm{G}$.

Los datos de la Sutel también revelan que el incremento de los suscriptores al servicio de Internet móvil, entre el primer trimestre del 2012 y el segundo del 2013, fue de un 86\%. Pasó de 1.893 .698 servicios activos a 3.521.251 en este periodo (Cordero, 2014, mayo 25, parra. 2-5).

La conectividad permanente y móvil a través de teléfonos inteligentes y tabletas ha traído a primera fila a las aplicaciones, también conocidas como "apps" por el diminutivo de su nombre en inglés (“applications”). Según un estudio de la corporación Oracle (2014), que llama a los millennials la "generación de las apps" (“the app generation”):

\begin{abstract}
No se puede negar la relevancia de las aplicaciones móviles para la población de hoy. La adopción de los teléfonos inteligentes y las tablets en todo el mundo sigue a todo vapor: sólo el año pasado [2013] 1.2 mil millones de teléfonos inteligentes fueron vendidos, un aumento del 23 por ciento en comparación con el año anterior. En paralelo, una explosión en el desarrollo de aplicaciones ha empoderado a las personas para sacarle el mayor provecho a estos dispositivos (parra. 1$)^{2}$.
\end{abstract}

El tema de la conectividad móvil ha adquirido tal importancia que, en el año 2013, salió a la luz una nueva revista académica de la prestigiosa casa editorial Sage: Medios Móviles y Comunicación ("Mobile Media and Communication"). En un artículo del primer número, Campbell (2013) sostiene que "la nueva revista representa un paso adelante en el desarrollo de la comunicación móvil como un campo de estudios" (parra. 1)3. Para él, el campo ha logrado posicionarse a través de otras iniciativas como conferencias, simposios, libros editados, listas de interés y centros de investigación. Aun así, el autor hace una reflexión crítica sobre si debería de haber un campo de estudio para la investigación y la teoría de los medios móviles y comunicación. Su conclusión es que sí, se trata de un campo de estudio legítimo que está "integralmente conectado al estudio de los medios y la comunicación" (Campbell, 2013, p. 12)

De esta misma revista académica destacan, por su temática, los trabajos de Martin (2014) y de Yamamoto et al. (2015). Ambos estudios parten de la creciente importancia que tienen los medios móviles, pero se enfocan también en la población de jóvenes y el tema de política y participación política. Para Martin (2014) "la popularidad y la importancia de los medios móviles como formas de acceder a la información política y participar en la política y las elecciones en todo el mundo ha crecido en años recientes" (p. 173) ${ }^{5}$. Yamamoto et al. (2014) citan estudios (Rainie et al., 2012; Smith and Duggan, 2012a, 2012b) sobre el crecimiento del uso de las redes sociales y las aplicaciones móviles, particularmente entre adultos jóvenes. Señalan que "de importancia para este grupo de edad es el aumento en el uso de las redes sociales y dispositivos móviles para obtener información política, la creación de contenido político generado por el usuario y la expresión de puntos de vista políticos y opiniones" (Yamamoto et al., 2014, p. 881) ${ }^{6}$.

2 There's no denying the relevance of mobile apps to today's population. Smartphone, tablet, and phablet adoption around the world continues to pick up steam - last year alone 1.2 billion smartphones were sold, a 23 percent rise compared to the year before In parallel, an explosion in the development of apps is empowering people to make the most of these devices.

3 This journal represents a step forward in the development of mobile communication studies as a field.

4 (...) integrally connected to the study of media and communication more broadly.

5 Mobile media have become increasingly popular and important in recent years as a means of accessing political information and participating in politics and elections worldwide.

6 Of significance for this age group is the rise in the use of social media and mobile devices for obtaining political information, creating user-generated political content, and expressing political views and opinions. 
Se trata, entonces, y sin lugar a dudas, no sólo de un fenómeno importante y permanente, sino también de uno que ya ha sido reconocido por la academia y que cuenta con por lo menos decenas de estudios que profundizan en su comprensión, teorizan y crean conocimiento nuevo sobre el fenómeno.

El muy reconocido Centro Pew de Investigación (Pew Research Center) publicó en abril 2015 un estudio que permite agregarle una variante más a la discusión. Se trata del uso de las encuestas a través de aplicaciones en los teléfonos inteligentes:

El auge de las aplicaciones en un mundo cada vez más móvil es una gran historia social, política y económica que [en el Pew Research Center] hemos estado documentando desde hace años. Como investigadores, hemos sentido desde hace tiempo que las aplicaciones son una gran promesa para la recolección de datos. No sólo podrían las encuestas basadas en aplicaciones, potencialmente, hacer a las encuestas más "amigablemente móviles", pero también podrían permitir a la gente proporcionar diferentes tipos de información que a veces es difícil reunir en las encuestas telefónicas tradicionales, tales como datos de localización o exactamente cuando las personas utilizan ciertas características de sus teléfonos inteligentes (McGeeney, 2015, abril 2, parra. 2) ${ }^{7}$.

Incluso ya desde el año 2010 (una eternidad cuando de tecnología móvil se trata), Brian F. Schaffner, politólogo de la Universidad de Massachusetts Amherst (2010, octubre 20), afirmó que las encuestas políticas en los Estados Unidos estaban experimentando cambios importantes debido a la creciente popularidad de los teléfonos celulares y el número cada vez menor de los estadounidenses con líneas telefónicas fijas tradicionales. En aquel momento, Schaffner estimó que entre el 35 y el 40 por ciento de los estadounidenses eran muy difíciles o imposibles de alcanzar en esos teléfonos fijos tradicionales y eran demográficamente particulares: "las personas que pueden ser alcanzadas por las líneas de tierra tienden a ser mayores, tienen familias, y están más conectados con sus comunidades 8 ." (UMass Amherst, 2010, octubre 20, parra. 3). El politólogo también señaló que otro elemento clave era el creciente número de personas, entre ellos muchos jóvenes, que utilizaban solamente los teléfonos celulares (UMass Amherst, 2010, octubre 20, parra. 4).

\section{Encuestas, valiosas pero con limitaciones}

Las encuestas y los sondeos tradicionales son resultado de procesos complejos, lentos y onerosos: debe definirse la cobertura geográfica, el tipo de muestreo, el tamaño de la muestra; luego, debe seleccionarse la muestra, contratar y entrenar a los encuestadores, asegurar controles de calidad, construir el instrumento, validarlo, salir al campo a realizar las entrevistas, procesar miles de datos y generar un informe.

A todo eso, hay que sumarle, además, el tiempo que tarde el medio que le dará difusión en preparar la o las notas para hacerlo y que entre en el ciclo noticioso (24 horas, en el caso de un diario). Así, cuando una encuesta se hace pública, los electores están frente a "una fotografía" de un momento del pasado.

Frente a una realidad caracterizada por la hiper-conectividad, con información y opiniones políticas al alcance de un clic, la opinión pública se mueve de forma vertiginosa y, también, altamente cambiante. En este contexto, las encuestas probablemente reflejen bien ese momento del pasado en el que se recabaron los datos, pero tienen mayores dificultades para explicar el presente y más aún para predecir tendencias futuras.

7 The rise of apps in an increasingly mobile world is a major social, political and economic story that we have been documenting for years. As researchers, we've felt for some time that apps hold a lot of promise for data collection. Not only could apps-based surveys potentially make surveys more "mobile friendly," but they might also allow people to provide different kinds of information that is sometimes difficult to gather in traditional phone surveys, such as location data or exactly when people use certain smartphone features. 
Pablo Fonseca, periodista experto en tecnología del diario La Nación y quien firma la nota de ese diario del 3 de febrero sobre cómo las redes sociales anunciaron el despegue de Luis Guillermo Solís en intención de voto (Fonseca, 2014 febrero 3), afirmó en esa misma publicación:

Las encuestas nunca hubieran podido mostrar esto por su metodología. Se dura días entre empezar la recolección de datos y su publicación. En el contexto actual, es cuestión de un día para que suceda una variación de relativa importancia. Una semana y todo puede cambiar por completo.

En cambio, esa variación diaria se ve más fácilmente si se analiza Google Trends o el big data de Twitter y Facebook. Tanto es así, que yo me atrevería a decir que sería posible calcular cuántos días más de campaña necesitaba Solís para ganar la elección en primera ronda.

\begin{abstract}
Al menos en esta campaña, hubo un cambio de intención de voto que varió mucho de un día para otro. Cambios tan veloces son muy difíciles de leer en encuestas, aunque es cierto que veíamos una tendencia de crecimiento de Solís en los últimos estudios de este tipo. No obstante, ante la mayor penetración de Internet y uso de redes sociales, parece ser más sencillo leer esos cambios de interés en esta otra fuente, al menos como una tendencia (parra. 11-13).
\end{abstract}

No se trata aquí de satanizar a las encuestas, pero sí de reconocer que no se le puede cerrar la puerta a un futuro que ya está aquí. La reflexión en torno al tema es impostergable, incluyendo evaluar si las encuestas son o no el mejor instrumento, o si deben ser el único instrumento autorizado por el TSE, de medición de intención de voto de las campañas electorales en el siglo XXI. En un artículo sobre el tema, Iñigo Lejarza, Gerente de Inteligencia de Medios de Grupo Nación, lo planteó así:

\begin{abstract}
En todo caso, la mala experiencia que hayamos podido tener, y el mal sabor de boca que nos hayan podido dejar las encuestas en un proceso electoral atípico desde muchos puntos de vista, no justifica prescindir de una herramienta que ha sido, es y será valiosa para muchos propósitos, aun cuando entre ellos no esté el predecir el nombre del próximo presidente de la República. Al satanizar las encuestas, corremos el riesgo de tirar al bebé con la bañera (Lejarza, 2014 febrero 9, parra. 10).
\end{abstract}

\title{
EI TSE no se puede quedar de brazos cruzados
}

Es inobjetable: el nuevo contexto obliga, sí o sí, a reconsiderar seriamente los conceptos sobre la política y la vida política y, por ende, las leyes y reglamentos que regulan los procesos electorales y, en este caso, la participación ciudadana en épocas de campaña electoral.

Bien lo expresó el analista político Víctor Ramírez (2014, abril 26): “con la lectura adecuada del extraordinario laboratorio político, sociológico y psicológico que fue esta última elección, los nuevos diputados están obligados a poner nuestra legislación electoral a la altura de la cultura cívica y democrática del pueblo costarricense" (parra. 12).

La ciudadanía de hoy es una más informada y crítica, con acceso a internet y redes sociales que han cambiado la manera como somos ciudadanos. Las generaciones de jóvenes, especialmente la conocida como los "millenials" y las que le siguen, son nativos digitales para quienes sus teléfonos inteligentes y las "apps" que a través de ellos utilizan son parte de su vida cotidiana, casi una extensión de su ser. No solamente se creó una nueva revista académica para publicar sobre investigaciones que precisamente estudian esta comunicación móvil (Campbell, 2013), sino que ya hay trabajos como los de Martin (2014) y Yamamoto et al. (2015) que discuten las relaciones entre medios móviles, política y participación política.

En Costa Rica, estudios académicos como el de Cruz Romero (2015) que exploran la capacidad predictiva de las redes sociales como herramienta de uso político electoral y publicaciones como las del 
periódico La Nación en plena campaña electoral (días antes de las elecciones) dan cuenta de que se está frente a una corriente que no dará marcha atrás.

Se trata de una realidad que ya ni siquiera es nueva y que obliga a todas las instituciones, incluyendo al TSE, a modernizarse y reformar reglamentos y normativa en consecuencia. Toda decisión que facilite la participación ciudadana servirá para fortalecer nuestro sistema político y la vida en democracia.

En esta ocasión, el TSE optó por cerrarle la puerta a la innovación y dejar vigentes las normas del pasado que consideran, únicamente, a las encuestas y sondeos tradicionales. Es importante que el caso de lo que sucedió con MiVotoHoy se conozca porque fue víctima en el pasado ciclo electoral. Lo que aquí se presenta debe servir para la reflexión y, ojalá, para reconocer la nueva realidad e impulsar el cambio, que ya es tardío. Se espera que iniciativas como MiVotoHoy no vuelvan a ser víctimas y, como país y como democracia, Costa Rica esté a la altura de los tiempos en las próximas elecciones.

\section{Bibliografía}

Alcoceba Hernando, José Antonio. (2013). Juventud, tecnologías de la información y cambio social. Perspectivas y escenarios para la socialización y la participación. En Ciudadanía, Tecnología y Cultura. Nodos conceptuales para pensar la nueva mediación digital. (Sierra Caballero, F. (coord.). Barcelona: Gedisa, Serie Comunicación Latina. 181-209.

Araya Alvarado, Sergio. (2014, febrero 4). Análisis: una mirada al proceso electoral de Costa Rica. En El Financiero. Recuperado de http://www.elfinancierocr.com/opinion/mirada-proceso-electoralCosta-Rica_0_458954113.html

Bernete García, Francisco. (2013). Identidades y mediadores de la ciudadanía digital. En Ciudadanía, Tecnología y Cultura. Nodos conceptuales para pensar la nueva mediación digital. (Sierra Caballero, F. (coord..). Barcelona: Gedisa, Serie Comunicación Latina. 151-179.

Campbell, Scott. (2013). Mobile Media and Communication: A New Field or Just a New Journal? En Mobile Media \& Communication. Enero. 1, No. 1, pp. 8-13.

Cambronero, Cristian. (2014, febrero 4). Las encuestas ya fueron. Blog fusildechispas.com. Recuperado de http://www.fusildechispas.com/2014/02/las-encuestas-ya-fueron/

Centro de Investigación en Estudios Políticos CIEP (2014). Informe de la encuesta post-electoral febrero 2014. Estudios de opinión socio-política. Universidad de Costa Rica. Recuperado de http://www.ciep.ucr.ac.cr/index.php/publicaciones/estudios-opinion/ Informe-de-la-Encuesta-Postelectoral-de-Febrero-del-2014/

Cordero, Mónica. (2014, mayo 25). Smartphones disparan conexiones a Internet en Costa Rica. En El Financiero. Recuperado de http://www.elfinancierocr.com/tecnologia/Internet_movil_Internet_fijo-Sutel-smartphones_0_523747660.html

Cruz Romero, Roberto. (2015). Política 2.0: Las TIC en la campaña electoral presidencial 2014 en Costa Rica. En PROSIC, Hacia la sociedad de la información y el conocimiento, 2014 (295-331). San José: PROSIC.

Fonseca, Pablo. (2014, febrero 3). Google, Twitter y Facebook anunciaron despegue de Luis Guillermo Solís en intención de voto. En La Nación. Recuperado de http://www.nacion.com/blogs/cazador_de_software/Google-Twitter-Facebook-Guillermo-Solis_10_1394560532.html

Fry, Richard. (2016, abril 25). Millenials Overtake Baby-Boomers as America's Largest Generation. En Pew Research Center. Recuperado de http://www.pewresearch.org/fact-tank/2016/04/25/ millennials-overtake-baby-boomers/

Katz, Josh. (2016, noviembre 8). Who Will Be President? En The New York Times. Recuperado de http:// www.nytimes.com/interactive/2016/upshot/presidential-polls-forecast.html 
Kristof, Nicholas. (2016, marzo 26). My Shared Shame: The Media Helped Make Trump. En The New York Times. Recuperado de http://www.nytimes.com/2016/03/27/opinion/sunday/my-sharedshame-the-media-helped-make-trump.html?src=me\&_r=1

Lara, Juan Fernando. (2014, enero 24). En Facebook, Luis Guillermo Solís genera más movimiento comparado a rivales. En La Nación. Recuperado de http://www.nacion.com/nacional/politica/ Facebook-Solis-movimiento-comparado-rivales_0_1392460782.html

(2014, febrero 4). Redes sociales anunciaron cómo se resolvió elección presidencial en Costa Rica. En La Nación. Recuperado de http://www.nacion.com/nacional/politica/Redes-sociales-adivinaroneleccion-presidencial_0_1394660569.html

Martin. Jason. (2014). Mobile news use and participation in elections: A bridge for the democratic divide? En Mobile Media \& Communication. Mayo, Vol. ,2 No. 2. 173-195 doi: $10.1177 / 2050157914520847$

McGeeney, Kyley. (2015, Abril 2). What We Learned About Surveying With Mobile Apps. En Pew Research Center. Recuperado de http://www.pewresearch.org/fact-tank/2015/04/02/ what-we-learned-about-surveying-with-mobile-apps/

Murillo, Álvaro. (2016, enero 13). Las elecciones municipales ligan más a los millennials. En Semanario Universidad. Recuperado de http://semanariouniversidad.ucr.cr/pais/ las-elecciones-municipales-ligan-mas-a-los-millennials/

National Public Radio. (2016, marzo 9). Why Did The Polls Fail To Predict Sanders' Win In Michigan? En NPR All Things Considered. Recuperado de http://www.npr.org/2016/03/09/469837001/ why-did-the-polls-fail-to-predict-sanders-win-in-michigan

Nielsen. (2014, septiembre 5). Mobile Millennials: Over 85\% of Generation Y Owns Smartphones. Recuperado de http:/www.nielsen.com/us/en/insights/news/2014/mobile-millennials-over85-percent-of-generation-y-owns-smartphones.html

Oracle Corporation. (2014). Millennials and Mobility: How Businesses Can Tap Into the App Generation. Recuperado de http://www.oracle.com/us/dm/millennials-and-mobility-2508385.pdf

Ramírez, Víctor. (2014, abril 25). Sobre productos y consumidores electorales. En La Nación. Recuperado de http://www.nacion.com/opinion/foros/productos-consumidores-electorales_0_1410658936.html

Rainie, Lee; Smith, Aaron y Schlozman, Kay Lehman, et al. (2012) Social media and political engagement. En Pew Internet \& American Life Project. Recuperado de http://pewinternet.org/ /media// Files/Reports/2012/PIP_SocialMediaAndPoliticalEngagement_PDF.pdf

Rueda Ortiz, Rocío. (2009). Convergencia tecnológica: síntesis o multiplicidad política y cultural. En Signo y Pensamiento, vol. XXVIII, núm. 54, enero-junio, pp. 114-130.

Ruiz Vega, Carolina. (2013, septiembre 21). Nueva app recopila intención de voto de usuarios de iPhone y de celulares con Android. En La Nación. Recuperado de http://www.nacion.com/tecnologia/ apps/Nueva-recopila-intencion-celulares-Android_0_1367463355.html

Rutenberg, Jim. (2016, mayo 5). The Republican Horse Race is Over, and Journalism Lost. En The New York Times. Recuperado de http://www.nytimes.com/2016/05/06/business/media/the-republicanhorse-race-is-over-and-journalism-lost.html?hp\&action=click\&pgtype=Homepage \&clickSource =story-heading\&module=first-column-region\&region=top-news\&WT.nav=top-news\&_r=1

Scarry, Eddie. (2016, marzo 5). How Donald Trump Played the Media. En Washington Examiner. Recuperado de http://www.washingtonexaminer.com/how-donald-trump-played-the-media/ article/2584764

Sierra Caballero, Francisco. (2013). Ciudadanía, Tecnología y Cultura. Nodos conceptuales para pensar la nueva mediación digital. Barcelona: Gedisa, Serie Comunicación Latina, pp. 17-57.

Smith, Aaron y Duggan, Maeve. (2012a) Online political videos and campaign 2012. En Pew Internet \& American Life Project. Recuperado de http://pewinternet.org/Reports/2012/Election-2012Video.aspx 
Smith, Aaron y Duggan, Maeve. (2012b) The state of the 2012 election - mobile politics. Pew Internet \& American Life Project. Recuperado de http://pewinternet.org/Reports/2012/Election-2012Mobile.aspx

Sobrado, Luis Antonio. (2014, abril 6). Tribunal Supremo de Elecciones. Discurso sesión solemne del 6 de abril de 2014. Recuperado de http://www.tse.go.cr/pdf/varios/sesion_solemne_abril2014.pdf

Tribunal Supremo de Elecciones. (2009). Reglamento sobre la inscripción para la realización de encuestas y sondeos de opinión de carácter político-electoral (Decreto №. 18-2009). Recuperado de https://www.tse.go.cr/pdf/normativa/inscripcionrealizacionencuestasysondeos.pdf

-(2013, agosto 29). Carta de la Dirección General del Registro Electoral y Financiamiento de Partidos Políticos al Secretario General del TSE. Oficio DGRE-400-2013.

-(2013, septiembre 5). Carta a Cristian Cambronero. Oficio DGRE 429-2013.

-(2015, noviembre 18). Comunicado de prensa. Mitad de electores costarricenses tienen entre 18 y 39 años. Recuperado de http://tse.go.cr/comunicado305.htm

Ulibarri, Eduardo. (2016, mayo 8). Trump y la ruptura electoral. En La Nación. Recuperdo de http:// www.nacion.com/opinion/foros/Trump-ruptura-electoral_0_1559444065.html

UMass Amherst (2010, octubre 20). UMass Amherst's Brian Schaffner Says Political Polls Changing as Cell Phones Proliferate and Land Lines Disappear. Recuperado de https://www.umass.edu/ newsoffice/article/umass-amherst $\%$ E2\%80\%99s-brian-schaffner-says-political-polls-changingcell-phones-proliferate-and

Yamamoto, Masahiro; Kushin, Mathew, y Dalisay, Francis. (2015). Social media and mobiles as political mobilization forces for young adults: Examining the moderating role of online political expression in political participation. En New Media \& Society. Junio, Vol. 17 No. 6. pp. 880-898 doi: $10.1177 / 1461444813518390$ 
